\title{
Generalized Exponential Bidirectional Fuzzy Associative Memory with Fuzzy Cardinality-Based Similarity Measures Applied to Face Recognition
}

\author{
A.C. SOUZA* and M.E. VALLE \\ Received on April 12, 2017 / Accepted on November 27, 2017
}

\begin{abstract}
Associative memories are biologically inspired models designed for the storage and recall by association. Such models aim to store a finite set of associations, called the fundamental memory set. The generalized exponential bidirectional fuzzy associative memory (GEB-FAM) is a heteroassociative memory model designed for the storage and recall of fuzzy sets. A similarity measure, that is, a function that indicates how much two fuzzy sets are equal, is at the core of a GEB-FAM model. In this paper, we present a detailed study on the use of cardinality-based similarity measures in the definition of a GEB-FAM. Moreover, we evaluate the performance of the GEB-FAMs defined using such measures in a face recognition problem.
\end{abstract}

Keywords: Associative memory, fuzzy set theory, similarity measure, face recognition.

\section{INTRODUCTION}

Associative memories are biologically inspired models aimed at storing and recalling information by association $[18,11,13]$. These models are designed for the storage of a finite set of association pairs $\left\{\left(\mathbf{a}^{1}, \mathbf{b}^{1}\right), \ldots,\left(\mathbf{a}^{p}, \mathbf{b}^{p}\right)\right\}$, called the fundamental memory set. Each association pair $\left(\mathbf{a}^{\xi}, \mathbf{b}^{\xi}\right)$ is a fundamental memory. Given an input pattern $\mathbf{a}^{\xi}$, the memory is expected to produce $\mathbf{b}^{\xi}$, or a sufficiently close pattern, as output. Also, the memory should be tolerant to noise and be able to retrieve an stored item $\mathbf{b}^{\xi}$ even from a corrupted version $\tilde{\mathbf{a}}^{\xi}$ of an original item $\mathbf{a}^{\xi}$. We speak of an autoassociative memory if $\mathbf{a}^{\xi}=\mathbf{b}^{\xi}$, for all $\xi=1, \ldots, p$. In this case, the fundamental memory set can be written as $\left\{\mathbf{a}^{1}, \ldots, \mathbf{a}^{p}\right\}$. If there is one $\xi \in\{1, \ldots, p\}$ such that $\mathbf{a}^{\xi} \neq \mathbf{b}^{\xi}$, the memory is said heteroassociative.

The Hopfield neural network, proposed by J. Hopfield in 1982, is a widely known neural network model able to implement an autoassociative memory for the storage of binary or bipolar vectors $[11,13,15]$. Despite its various applications [14, 26, 25], the Hopfield neural network suffers from a low absolute storage capacity [23]. Such limitation motivated many researchers to

*Corresponding author: Aline C. Souza - E-mail: s.alinedesouza@ gmail.com

Departamento de Matemática Aplicada, Universidade Estadual de Campinas, 13083-859 Campinas, SP, Brasil. E-mail: s.alinedesouza@gmail.com; valle@ime.unicamp.br 
develop improved versions of the Hopfield neural network [12, 10, 9]. In particular, Chiueh and Goodman introduced the exponential correlation associative memory (ECAM), a high-capacity autoassociative model designed for the storage of bipolar patterns [3]. For the heteroassociative case, Jeng et al. proposed the exponential bidirectional associative memory (EBAM) model that can be used to store pairs of bipolar vectors [16].

Many applications of associative memories, however, require storage of vectors with real components or fuzzy sets $[1,2,7,6,19,29,32]$. The generalized recurrent exponential fuzzy associative memories $[28,29]$ (GRE-FAMs) are designed for the storage and recall of a finite family of fuzzy sets. Furthermore, the GRE-FAMs corresponds to a fuzzy version of the ECAM model of Chiueh and Goodman. Inspired by the EBAM, we recently introduced the generalized exponential bidirectional fuzzy associative memories (GEB-FAMs), an extension of GRE-FAMs for the heteroassociative case [27].

Like the GRE-FAMs, GEB-FAMs use a similarity measure in their definition. In general terms, a similarity measure is a function that indicates how much two fuzzy sets are equal. In previous works, we considered a normalized version of the similarity measure proposed by Xuecheng [30]. In this work, however, we adopt a more general definition proposed by De Baets and De Meyer [4] and focus on cardinality-based similarity measures [5, 4]. Furthermore, we performed extensive computational experiments in order to evaluate the performance of GEB-FAMs based on these similarity measures in a face recognition problem.

This work is organized as follows. In the next section, we review the definition of fuzzy similarity measures proposed by De Baets and De Meyer and list some cardinality-based similarity measures [5, 4]. The GEB-FAMs are presented in Section 3. Computational experiments, performed to evaluate the performance of GEB-FAMs in a face recognition problem, are described and analyzed in Section 4. We finish the paper with the concluding remarks in Section 5.

\section{FUZZY SETS AND SIMILARITY MEASURES}

We begin this section by recalling the definition of fuzzy sets [31]. Subsequently, we present some cardinality-based fuzzy similarity measures proposed by De Baets and De Meyer in [4].

A fuzzy set $A$ on an universe of discourse $U$ is characterized by its membership function $A: U \rightarrow$ $[0,1]$. This function indicates the membership degree of each element $u \in U$ to the fuzzy subset $A$. Thus, $A(u)=0$ means that $u$ does not belong to $A$ and $A(u)=1$ means total membership of $u$ to $A$. We denote by $\mathscr{F}(U)$ the family of all fuzzy sets of $U$.

Throughout this paper, we only consider fuzzy sets on a finite universe of discourse $U=$ $\left\{u_{1}, \ldots, u_{n}\right\}$. In this case, we can identify a fuzzy set $A$ with a vector $A=\left(A\left(u_{1}\right), \ldots, A\left(u_{n}\right)\right) \in$ $[0,1]^{n}[20]$. The cardinality and the complement of a fuzzy set $A$ on a finite universe of discourse $U=\left\{u_{1}, \ldots, u_{n}\right\}$ are defined respectively by

$$
\# A=\sum_{i=1}^{n} A\left(u_{i}\right) \quad \text { and } \quad A^{c}\left(u_{i}\right)=1-A\left(u_{i}\right), \forall i=1, \ldots, n .
$$


A triangular norm, t-norm for short, is an increasing, commutative, and associative mapping $T:[0,1] \times[0,1] \rightarrow[0,1]$ with neutral element 1 , that is, $T(1, x)=T(x, 1)=x$ for all $x \in[0,1]$ [17]. The intersection $A \cap B$ and the union $A \cup B$ of two fuzzy sets $A, B \in \mathscr{F}(U)$ can be computed as follows using a t-norm:

$$
(A \cap B)\left(u_{i}\right)=T\left(A\left(u_{i}\right), B\left(u_{i}\right)\right), \quad \forall i=1, \ldots, n,
$$

and

$$
(A \cup B)\left(u_{i}\right)=A\left(u_{i}\right)+B\left(u_{i}\right)-T\left(A\left(u_{i}\right), B\left(u_{i}\right)\right), \quad \forall i=1, \ldots, n .
$$

Furthermore, the difference $A \backslash B$ and the symmetric difference $A \Delta B$ of fuzzy sets $A$ and $B$ are respectively defined by

$$
(A \backslash B)\left(u_{i}\right)=A\left(u_{i}\right)-T\left(A\left(u_{i}\right), B\left(u_{i}\right)\right), \quad \forall i=1, \ldots, n,
$$

and

$$
(A \Delta B)\left(u_{i}\right)=A\left(u_{i}\right)+B\left(u_{i}\right)-2 T\left(A\left(u_{i}\right), B\left(u_{i}\right)\right), \quad \forall i=1, \ldots, n .
$$

We would like to point out that the identity $\#(A \cup B)+\#(A \cap B)=(\# A)+(\# B)$ holds true if the intersection is modeled using a Frank t-norm [8, 24]. A Frank t-norm, denoted by $T_{s}, s \in[0, \infty]$, is defined by

$$
T_{s}(x, y)=\log _{s}\left[1+\frac{\left(s^{x}-1\right)\left(s^{y}-1\right)}{s-1}\right], \quad s>0, s \neq 1,
$$

with limiting values:

(i) $T_{0}(x, y)=\lim _{s \rightarrow 0} T_{s}(x, y)=M(x, y)=\min \{x, y\}$,

(ii) $T_{1}(x, y)=\lim _{s \rightarrow 1} T_{s}(x, y)=P(x, y)=x y$,

(iii) $T_{\infty}(x, y)=\lim _{s \rightarrow \infty} T_{s}(x, y)=W(x, y)=\max \{x+y-1,0\}$.

Besides Frank's t-norms, in this paper we also consider the drastic product t-norm $Z:[0,1] \times$ $[0,1] \rightarrow[0,1]$ defined by

$$
Z(x, y)= \begin{cases}1, & x=1 \text { or } y=1 \\ 0, & \text { otherwise }\end{cases}
$$

A fuzzy similarity measure is a function that associates to a given pair of fuzzy sets a real number on the interval $[0,1]$ that indicates the degree of equality of these fuzzy sets. The definition of a fuzzy similarity measure may vary according to the context. In our previous works [28, 29], we adopted a normalized version of the axiomatic definition proposed by Xuecheng [30]. In this work, we consider a more general definition proposed by De Baets and De Meyer in [4]. According to this definition, a fuzzy similarity measure is a symmetric binary fuzzy relation on $\mathscr{F}(U)$, i.e., a symmetric function $\mathscr{S}: \mathscr{F}(U) \times \mathscr{F}(U) \rightarrow[0,1]$. 
We speak of a reflexive similarity measure $S: \mathscr{F}(U) \times \mathscr{F}(U) \rightarrow[0,1]$ if the identity $S(A, A)=1$ holds for any fuzzy set $A \in \mathscr{F}(U)$ [4]. Analogously, a similarity measure is said to be locally reflexive if $S(A, A) \geq S(A, B)$ for all $A, B \in \mathscr{F}(U)$. Finally, given a t-norm $T$, we say that $S$ is $T$-transitive if the inequality

$$
T(S(A, B), S(B, C)) \leq S(A, C)
$$

holds true for all fuzzy sets $A, B, C \in \mathscr{F}(U)$.

In [4], De Beats and De Meyer introduced the following class of rational similarity measures based on the cardinality of fuzzy sets on a finite universe of discourse:

$$
S(A, B)=\frac{a \alpha_{A, B}+b \omega_{A, B}+c \delta_{A, B}+d v_{A, B}}{a^{\prime} \alpha_{A, B}+b^{\prime} \omega_{A, B}+c^{\prime} \delta_{A, B}+d^{\prime} v_{A, B}} .
$$

where $a, a^{\prime}, b, b^{\prime}, c, c^{\prime}, d, d^{\prime} \in\{0,1\}$ are parameters and

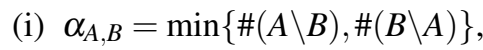

(ii) $\omega_{A, B}=\max \{\#(A \backslash B), \#(B \backslash A)\}$,

(iii) $\delta_{A, B}=\#(A \cap B)$,

(iv) $v_{A, B}=\#(A \cup B)^{c}$.

Table 1 shows some similarity measures derived from (2.5). Here, the fuzzification schemes described by (2.1), (2.2), (2.3), and (2.4) are all based on a Frank t-norm. In this paper, we focus on the similarity measures given by Table 1 with the minimum $T_{0}(x, u)=M(x, y)=\min (x, y)$, the algebraic product $T_{1}(x, y)=P(x, y)=x y$, and the Łukasiewicz t-norm $T_{\infty}(x, y)=W(x, y)=$ $\max \{x+y-1,0\}$.

We would like to conclude this section by pointing out that some similarity measures given by Table 1 satisfy the following properties [4]:

(i) $R_{i}$, for $i=1, \ldots, 15$, are all reflexive,

(ii) $S_{17}, S_{18}$, and $S_{19}$ are locally reflexive,

(iii) $R_{1}, R_{5}, R_{6}, R_{13}, R_{15}$, and $S_{18}$ are $W$-transitive,

(iv) $R_{11}$ and $R_{14}$ are $P$-transitive,

(v) $S_{19}$ is $M$-transitive,

(vi) $R_{2}$ is $Z$-transitive, 
Table 1: Expressions of rational cardinality-based similarity measures.

\begin{tabular}{cccc}
\hline $\mathrm{S}$ & Expression & $\mathrm{S}$ & Expression \\
\hline$R_{1}$ & $\frac{\#(A \cap B)}{\max \{\# A, \# B\}}$ & $R_{10}$ & $\frac{\min \{\#(A \backslash B), \#(B \backslash A)\}}{\max \{\#(A \backslash B), \#(B \backslash A)\}}$ \\
$R_{2}$ & $\frac{\#\left(A \Delta B c^{c}\right.}{\max \left\{\#(A \backslash B)^{c}, \#(B \backslash A)^{c}\right\}}$ & $R_{11}$ & $\frac{\min \{\# A, \# B\}}{\max \{\# A, \# B\}}$ \\
$R_{3}$ & $\frac{\#(A \cap B)}{\min \{\# A, \# B\}}$ & $R_{12}$ & $\frac{\min \left\{\#(A \backslash B)^{c}, \#(B \backslash A)^{c}\right\}}{\max \left\{\#(A \backslash B)^{c}, \#(B \backslash A)^{c}\right\}}$ \\
$R_{4}$ & $\frac{\#(A \Delta B)^{c}}{\min \left\{\#(A \backslash B)^{c}, \#(B \backslash A)^{c}\right\}}$ & $R_{13}$ & $\frac{\min \{\#(A \backslash B), \#(B \backslash A)\}}{\#(A \Delta B)}$ \\
$R_{5}$ & $\frac{\#(A \cap B)}{\#(A \cup B)}$ & $R_{14}$ & $\frac{\min \{\# A, \# B\}}{\#(A \cup B)}$ \\
$R_{6}$ & $\frac{\#(A \Delta B)^{c}}{n}$ & $R_{15}$ & $\frac{\min \left\{\#(A \backslash B)^{c}, \#(B \backslash A)^{c}\right\}}{n}$ \\
$R_{7}$ & $\frac{\max \{\#(A \backslash B), \#(B \backslash A)\}}{\#(A \Delta B)}$ & $S_{17}$ & $\frac{\#(A \cap B)}{\max \left\{\#(A \backslash B)^{c}, \#(B \backslash A)^{c}\right\}}$ \\
$R_{8}$ & $\frac{\max \{\# A, \# B\}}{\#(A \cup B)}$ & $S_{18}$ & $\frac{\#(A \cap B)}{n}$ \\
$R_{9}$ & $\frac{\max \left\{\#(A \backslash B)^{c}, \#(B \backslash A)^{c}\right\}}{n}$ & $S_{19}$ & $\frac{\min \{\# A, \# B\}}{n}$ \\
& & &
\end{tabular}

\section{GENERALIZED EXPONENTIAL BIDIRECTIONAL FUZZY ASSOCIATIVE MEMORIES}

In this section, we present the generalized exponential bidirectional fuzzy associative memories (GEB-FAMs), which have been recently proposed by us in the conference paper [27].

A GEB-FAM can be used for the storage and recall of a finite set of pairs of fuzzy sets. Formally, let $\left\{\left(A^{\xi}, B^{\xi}\right), \xi=1, \ldots, p\right\} \subset \mathscr{F}(U) \times \mathscr{F}(V)$ be the fundamental memory set, $\alpha>0$ a real number, $\mathscr{S}_{U}: \mathscr{F}(U) \times \mathscr{F}(U) \rightarrow[0,1]$ and $\mathscr{S}_{V}: \mathscr{F}(V) \times \mathscr{F}(V) \rightarrow[0,1]$ similarity measures, $G$ and $H$ real-valued $p \times p$ matrices. Given an input fuzzy set $X_{0} \in \mathscr{F}(U)$, a GEB-FAM produces recursively sequences of fuzzy sets $\left\{X_{t}\right\}_{t>0}$ and $\left\{Y_{t}\right\}_{t \geq 0}$ defined, for all $t \geq 0, u \in U$, and $v \in V$, as follows:

$$
Y_{t}(v)=\varphi\left(\frac{\sum_{\xi=1}^{p} \sum_{\mu=1}^{p} g_{\xi \mu} e^{\alpha \mathscr{S}_{U}\left(A^{\mu}, X_{t}\right)} B^{\xi}(v)}{\sum_{\eta=1}^{p} \sum_{\mu=1}^{p} g_{\eta \mu} e^{\alpha \mathscr{S}_{U}\left(A^{\mu, X_{t}}\right)}}\right)
$$


and

$$
X_{t+1}(u)=\varphi\left(\frac{\sum_{\xi=1}^{p} \sum_{\mu=1}^{p} h_{\xi \mu} e^{\alpha \mathscr{S}_{V}\left(B^{\mu}, Y_{t}\right)} A^{\xi}(u)}{\sum_{\eta=1}^{p} \sum_{\mu=1}^{p} h_{\eta \mu} e^{\alpha \mathscr{S}_{V}\left(B^{\mu, Y_{t}}\right)}}\right),
$$

where the function $\varphi$, given by

$$
\varphi(x)=\max (0, \min (1, x))
$$

ensures that $Y_{t}(v), X_{t+1}(u) \in[0,1]$ for all $v \in V, u \in U$, and $t>0$. In this work, we consider $G=C^{-1}$, where $C=\left(c_{i j}\right)_{p \times p}$ is the matrix defined by $c_{i j}=e^{\alpha \mathscr{S}_{U}\left(A^{i}, A^{j}\right)}$. Analogously, we can define $H=D^{-1}$, where $D=\left(d_{i j}\right)_{p \times p}$ is given by $d_{i j}=e^{\alpha \mathscr{S}_{V}\left(B^{i}, B^{j}\right)}$ for all $i, j=1, \ldots, p$.

\section{COMPUTATIONAL EXPERIMENTS}

In this section, we evaluate the performance of GEB-FAMs in a face recognition problem using the AR database [22]. Furthermore, we confront the GEB-FAM with the state-of-the-art approaches by performing the same experiment described by Luo et al. In fact, Table 2, extracted from [21], gives the accuracy obtained by several state-of-the-art approaches to the face recognition problem using the AR database as explained below.

Table 2: Accuracy obtained by several models applied to the face recognition problem using the

\begin{tabular}{|c|c|c|c|c|c|c|}
\hline & LRC & CRC & $\mathrm{SRC}$ & CESR & RSC & SSEC \\
\hline a) & 90.2 & 83.3 & 95.6 & 93.5 & 90.6 & 75.0 \\
\hline \multirow[t]{2}{*}{ b) } & 30.4 & 54.6 & 54.8 & 34.8 & 39.8 & 23.1 \\
\hline & \multicolumn{2}{|c|}{$\mathrm{SNL}_{2} \mathrm{R} 2$} & $\mathrm{SNL}_{1} \mathrm{R} 1$ & $\mathrm{DNL}_{2} \mathrm{R} 2$ & \multicolumn{2}{|c|}{$\mathrm{DNL}_{1} \mathrm{R} 1$} \\
\hline a) & \multicolumn{2}{|c|}{95.7} & 96.1 & 95.8 & \multicolumn{2}{|c|}{96.7} \\
\hline b) & \multicolumn{2}{|c|}{70.2} & 71.2 & 70.4 & \multicolumn{2}{|c|}{72.3} \\
\hline
\end{tabular}
AR database.

In the face recognition problem, we must identify a person from a face image using a set of labeled images, called the training set. Such as Luo et al., the gray-scale images from the AR database have been cropped and reshaped to dimension $50 \times 40$. We considered 8 gray-scale images of each individual from a group of 120 people as the training set. Figure 1 shows the 8 gray-scale images from a certain individual of the training set. Two experiments were conducted to evaluate the performance of an approach to the face recognition problem:

(a) A test set composed by 4 images from each individual with sunglasses and different illumination conditions. 


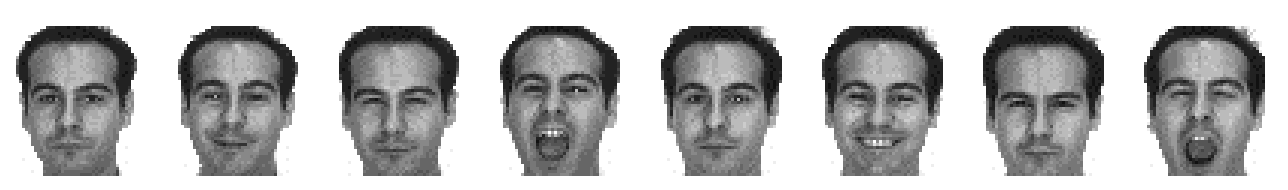

Figure 1: Images of an individual from the training set.

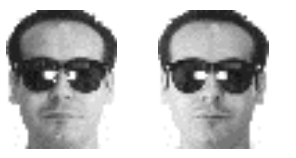

a) sunglasses+illumination.

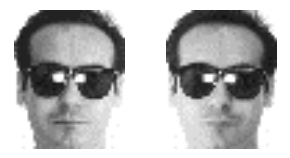

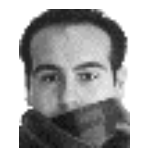
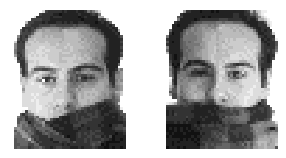

b) scarf+illumination.

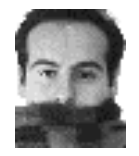

Figure 2: Images in the test set of a certain individual in the experiments.

(b) A test set consisting of 4 images from each individual with scarf and different illumination conditions.

Figure 2 shows the test images of the individual of Figure 1 in the experiments: a) sunglasses+illumination and b) scarf+illumination, respectively.

Let us now describe how a GEB-FAM can be applied for face recognition. First, we identified each face image with a fuzzy subset $A^{\xi}$, for $\xi=1, \ldots, 960$, on an universe of discourse $U=\{(1,1),(1,2), \ldots,(50,39),(50,40)\}$. Moreover, for each $\xi \in\{1, \ldots, 960\}$, we defined $B^{\xi}$ : $\{1,2, \ldots, 120\} \rightarrow\{0,1\}$ as follows:

$$
B^{\xi}(i)= \begin{cases}1, & A^{\xi} \text { belongs to the } i \text {-th person, } \\ 0, & \text { otherwise }\end{cases}
$$

Note that $B^{\xi}$ indicates which person the face image $A^{\xi}$ belongs to. Also, by taking $V=$ $\{1, \ldots, 120\}$, we have $B^{\xi} \in \mathscr{F}(V)$. Therefore, we can design GEB-FAMs for the storage of the fundamental memory set $\left\{\left(A^{\xi}, B^{\xi}\right), \xi=1, \ldots, 960\right\} \subset \mathscr{F}(U) \times \mathscr{F}(V)$. Then, given a test image $X_{0}$, we determine the fuzzy set produced by a GEB-FAM in a single step, i.e. we calculate $Y_{0}$ using (3.1) (with $t=0$ ). We associate the test image $X_{0}$ to the $i$-th person, where $i$ is the first index such that $Y_{0}(i) \geq Y_{0}(j)$, for all $j=1, \ldots, 120$.

It turns out that a GEB-FAM depends on the similarity measure $\mathscr{S}_{U}$ and the parameter $\alpha>0$. In our computational experiments, we considered all the similarity measures listed in Table 1. Precisely, for each fuzzy similarity measure on Table 1, we used the fuzzification schemes described in Section 2 with the minimum, the product, and the Łukasiewicz t-norm. As a consequence, we have 50 fuzzy similarity measures, namely, the three fuzzy versions of each of the similarity measures $R_{1}, R_{2}, \ldots, R_{10}, R_{12}, \ldots, R_{15}, S_{17}, S_{18}$, and the similarity measures $R_{11}$ and $S_{19}$, which 
do not depend on the t-norm but only on the cardinality of the involved fuzzy sets. In addition, for each one of these 50 similarity measures, we considered five values for the parameter $\alpha$, namely, $\alpha \in\{0.5,1,5,10,30\}$. Summarizing, the 50 similarity measures combined with the 5 values of the parameter $\alpha$ yielded a total of 250 different GEB-FAMs.

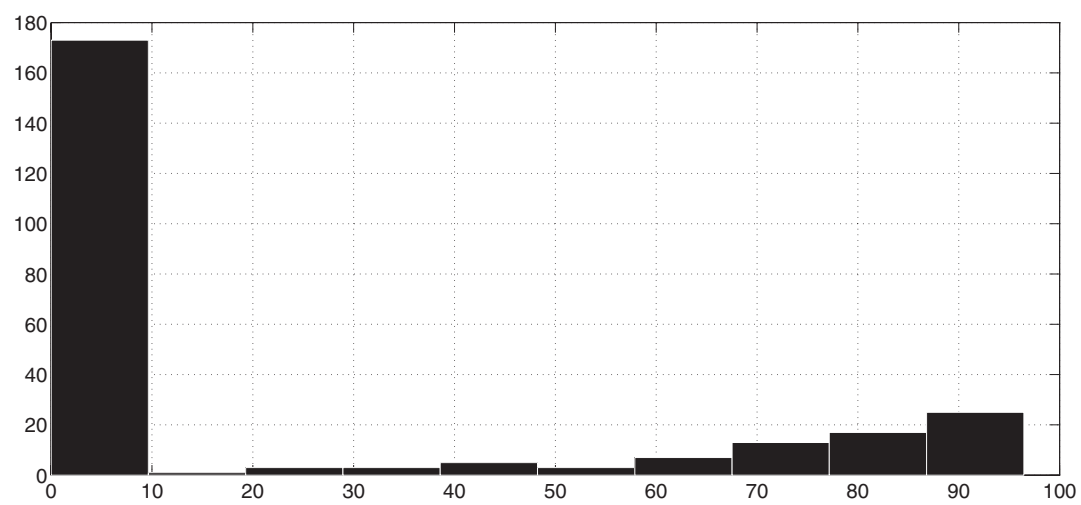

a) sunglasses+illumination

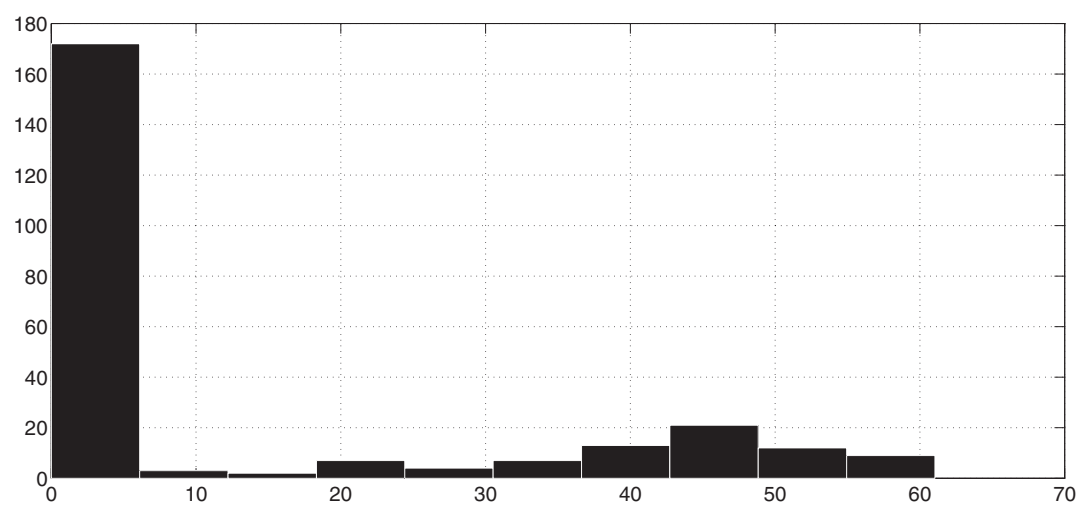

b) scarf+illumination

Figure 3: Histogram of the accuracy rates obtained by considering all 250 GEB-FAMs.

The accuracy rates obtained by all the 250 GEB-FAMs in both experiments are summarized in the histograms shown in Figure 3. Note that a large number of GEB-FAMs yielded an accuracy rate less than or equal to $10 \%$. By looking over at the poor recognition rates, most have been produced by a GEB-FAM based on a non T-transitive, with respect to the minimum, product, Łukasiewicz, or drastic product, fuzzy similarity measure. Precisely, except the memory based on $S_{17}$, the GEB-FAMs based on non T-transitive similarity measures yielded recognition rates below 10\%. The GEB-FAM based on the locally-reflexive non T-transitive similarity measure $S_{17}$ with the minimum and the product t-norms yielded accuracy rates of $94.58 \%$ (for $\alpha=30$ ) 
and $53.54 \%$ (for $\alpha=5$ ) in experiments a) and b), respectively. In the light of these remarks, let us focus on T-transitive similarity measures.

For each T-transitive similarity measure listed in Table 1, we selected the parameter $\alpha \in$ $\{0.5,1,5,10,30\}$ that produced the largest recognition rate. Figure 4 shows the boxplot of the accuracy values of the 24 fine-tuned GEB-FAM models by the fuzzification schemes.

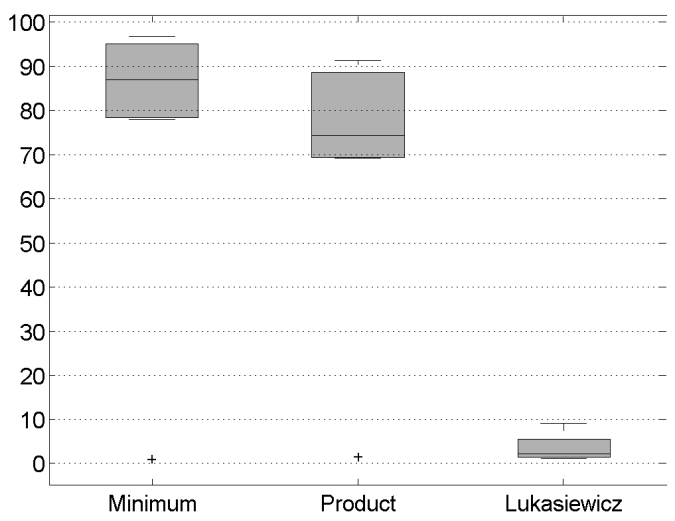

a) sunglasses+illumination

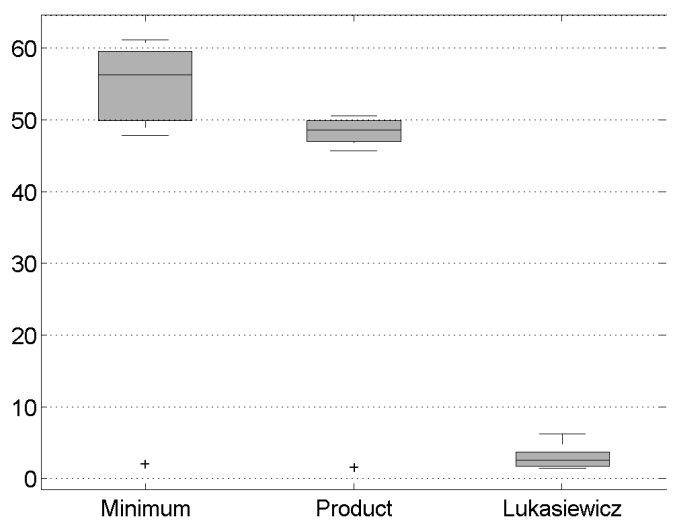

b) scarf+illumination

Figure 4: Boxplot of the accuracy rates produced by fine-tuned GEB-FAMs based on T-transitive similarity measures by the fuzzification scheme.

Precisely, the first boxplot refers to the best GEB-FAMs based on T-transitive similarity measures with the minimum in (2.1), (2.2), (2.3), and (2.4). Similarly, the second and the third boxplots correspond to the GEB-FAMs obtained by using respectively the product and Łucasievicz t-norm. According to the Figure 4, the GEB-FAMs obtained by considering the Łukasiewicz t-norm also yielded poor recognition rates. Excluding the GEB-FAM based on the similarity measure $R_{13}$, 
which correspond to the two outliers shown in the boxplots on Figure 4, the GEB-FAM models derived from the minimum and the product achieved performance competitive to other models from the literature.

\section{CONCLUDING REMARKS}

In this paper, we investigated the role of a fuzzy similarity measure in a generalized exponential bidirectional fuzzy associative memory (GEB-FAM). Precisely, we first revised the cardinalitybased similarity measures and the fuzzification schemes proposed by De Baets and De Meyer $[5,4]$. Then, we used these fuzzy similarity measures to design single-step GEB-FAM models for a face recognition task.

Using the AR database and 250 different GEB-FAM models, we concluded that the memories based on non T-transitive similarity measures usually produce poor performance, i.e., a recognition rates below $10 \%$. The only exception we found is the GEB-FAM based on the locally reflexive non T-transitive measure $S_{17}$ with minimum t-norm, which achieved an accuracy rates of $94.58 \%$ and $53.54 \%$ in experiments a) sunglasses+illumination and b) scarf+illumination, respectively. GEB-FAMs based on T-transitive similarity measures with the Łucasievicz t-norm also exhibit poor recognition rates. In contrast, GEB-FAMs defined using T-transitive measures with either minimum or product t-norms achieved competitive results in comparison with others models from literature. In particular, the best results in this face recognition problem were obtained by considering T-transitive fuzzy similarity measures based on the minimum t-norm.

\section{ACKNOWLEDGEMENTS}

This work was supported in part by FAPESP under grant no. 2015/00745-1 and by CNPq under grant no. 305486/2014-4.

RESUMO. Memórias associativas são modelos biologicamente inspirados projetados para o armazenamento e recordação por associação. Tais modelos visam o armazenamento de um conjunto finito de associações, chamado conjunto das memórias fundamentais. A memória associativa bidirecional exponencial fuzzy generalizada (GEB-FAM) é um modelo de memória heteroassociativa para armazenamento e recordação de conjuntos fuzzy. Uma medida de similaridade, isto é, uma função que indica o grau de igualdade entre dois conjuntos fuzzy, está no centro de um modelo GEB-FAM. Neste trabalho, apresentamos um estudo detalhado sobre o uso de medidades de similaridade definidas com base na cardinalidade na definição de uma GEB-FAM. Sobretudo, avaliamos o desempenho das GEB-FAMs usando tais medidas de similaridade em um problema de reconhecimento de faces.

Palavras-chave: Memória associativa, teoria dos conjuntos fuzzy, medida de similaridade, reconhecimento de face. 


\section{REFERENCES}

[1] M. Aldape-Pérez, C. Yáñez Márquez, O. Camacho-Nieto \& A. J.Argüelles-Cruz. An Associative Memory Approach to Medical Decision Support Systems. Computer Methods and Programs in Biomedicine, 106(3) (2012), 287-307. doi:10.1016/j.cmpb.2011.05.002.

[2] P. Binh \& N. Hoa. Compare Effective Fuzzy Associative Memories for Grey-Scale Image Recognition. In P. Vinh, N. Hung, N. Tung \& J. Suzuki (editors), "Context-Aware Systems and Applications", volume 109 of Lecture Notes of the Institute for Computer Sciences, Social Informatics and Telecommunications Engineering. Springer Berlin Heidelberg (2013), pp. 258-267. doi:10.1007/ 978-3-642-36642-0_26.

[3] T.D. Chiueh \& R.M. Goodman. Recurrent Correlation Associative Memories. IEEE Transactions on Neural Networks, 2(2) (1991), 275-284. doi:10.1109/72.80338.

[4] B. De Baets \& H. De Meyer. Transitivity-preserving fuzzification schemes for cardinality-based similarity measures. European Journal of Operational Research, 160(3) (2005), 726 - 740. doi:10.1016/ j.ejor.2003.06.036.

[5] B. De Baets, H. De Meyer \& H. Naessens. A class of rational cardinality-based similarity measures. Journal of Computational and Applied Mathematics, 132(1) (2001), 51-69.

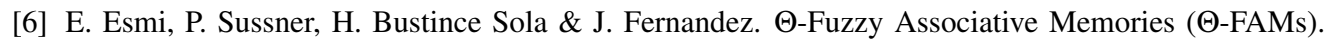
IEEE Transactions on Fuzzy Systems, 23(2) (2015), 313 - 326.

[7] E.L. Esmi, P. Sussner, M.E. Valle, F. Sakuray \& L. Barros. Fuzzy Associative Memories Based on Subsethood and Similarity Measures with Applications to Speaker Identification. In "Lecture Notes in Computer Science: International Conference on Hybrid Artificial Intelligence Systems (HAIS 2012)". Springer-Verlag Berlin Heidelberg, Berlin, Germany (2012), pp. 479-490.

[8] M.J. Frank. On the simultaneous associativity of $\mathrm{F}(\mathrm{x}, \mathrm{y})$ and $\mathrm{x}+\mathrm{y}-\mathrm{F}(\mathrm{x}, \mathrm{y})$. Aequationes Mathematicae, 19(1) (1979), 194-226. doi:10.1007/BF02189866.

[9] C. García \& J.A. Moreno. The Hopfield Associative Memory Network: Improving Performance with the Kernel "Trick". In "Lecture Notes in Artificial Inteligence - Proceedings of IBERAMIA 2004", volume 3315 of Advances in Artificial Intelligence - IBERAMIA 2004. Springer-Verlag (2004), pp. $871-880$.

[10] V. Giminez-Martinez. A Modified Hopfield Auto-Associative Memory with Improved Capacity. IEEE Transactions on Neural Networks, 11(4) (2000), 867-878.

[11] M.H. Hassoun \& P.B. Watta. Associative Memory Networks. In E. Fiesler \& R. Beale (editors), "Handbook of Neural Computation". Oxford University Press (1997), pp. C1.3:1-C1.3:14.

[12] M.H. Hassoun \& A.M. Youssef. A New Recording Algorithm for Hopfield Model Associative Memories. In "Neural Network Models for Optical Computing", volume 882 of Proceedings of SPIE (1988), pp. 62-70.

[13] R. Hecht-Nielsen. "Neurocomputing”. Addison-Wesley, Reading, MA (1989). 
[14] J. Hopfield \& D. Tank. Neural computation of decisions in optimization problems. Biological Cybernetics, 52 (1985), 141-152.

[15] J.J. Hopfield. Neural Networks and Physical Systems with Emergent Collective Computational Abilities. Proceedings of the National Academy of Sciences, 79 (1982), 2554-2558.

[16] Y.J. Jeng, C.C. Yeh \& T.D. Chiueh. Exponential bidirectional associative memories. Eletronics Letters, 26(11) (1990), 717-718. doi:10.1049/el:19900468.

[17] E.P. Klement, R. Mesiar \& E. Pap. Triangular norms. (2000).

[18] T. Kohonen. "Self-organization and associative memory". Springer-Verlag New York, Inc., New York, NY, USA, 2rd edition edition (1987).

[19] S.G. Kong \& B. Kosko. Adaptive Fuzzy Systems for Backing up a Truck-and-Trailer. IEEE Transactions on Neural Networks, 3(2) (1992), 211-223.

[20] B. Kosko. "Neural Networks and Fuzzy Systems: A Dynamical Systems Approach to Machine Intelligence". Prentice Hall, Englewood Cliffs, NJ (1992).

[21] L. Luo, J. Yang, J. Qian \& Y. Tai. Nuclear-L1 norm joint regression for face reconstruction and recognition with mixed noise. Pattern Recognition, 48(12) (2015), 3811 - 3824. doi:10.1016/j.patcog. 2015.06.012.

[22] A.M. Martinez. The AR face database. CVC Technical Report, 24 (1998). URL http://ci.nii. ac.jp/naid/10012519345/en/.

[23] R.J. McEliece, E.C. Posner, E.R. Rodemich \& S. Venkatesh. The capacity of the Hopfield associative memory. IEEE Transactions on Information Theory, 1 (1987), 33-45.

[24] H.T. Nguyen \& E.A. Walker. "A First Course in Fuzzy Logic”. Chapman \& Hall/CRC, Boca Raton, 2 edition (2000).

[25] G. Serpen. Hopfield Network as Static Optimizer: Learning the Weights and Eliminating the Guesswork. Neural Processing Letters, 27(1) (2008), 1-15. doi:10.1007/s11063-007-9055-8.

[26] K. Smith, M. Palaniswami \& M. Krishnamoorthy. Neural Techniques for Combinatorial Optimization with Applications. IEEE Transactions on Neural Networks, 9(6) (1998), 1301-1318.

[27] A.C. Souza \& M.E. Valle. Memória Associativa Bidirecional Exponencial Fuzzy Generalizada Aplicada ao Reconhecimento de Faces. In M.E.R. do Valle Mesquita; Graçaliz Pereira Dimuro; Regivan Hugo Nunes Santiago; Estevão Esmi Laureano (editor), "Recentes Avanços em Sistemas Fuzzy”, volume 1. Sociedade Brasileira de Matemática Aplicada e Computacional (SBMAC), São Carlos - SP (2016), pp. 503-514. ISBN: 978-85-8215-079-5.

[28] A.C. Souza, M.E. Valle \& P. Sussner. Generalized Recurrent Exponential Fuzzy Associative Memories Based on Similarity Measures. In "Proceedings of the 16th World Congress of the International Fuzzy Systems Association (IFSA) and the 9th Conference of the European Society for Fuzzy Logic and Technology (EUSFLAT)”, volume 1. Atlantis Press (2015), pp. 455-462. doi:10.2991/ ifsa-eusflat-15.2015.66. 
[29] M.E. Valle \& A.C. de Souza. "Pattern Classification using Generalized Recurrent Exponential Fuzzy Associative Memories”, volume 6. Science Gate Publishing (2016), chapter 4, pp. 79-102. doi:10. 15579/gcsr.vol6.ch4.

[30] L. Xuecheng. Entropy, distance measure and similarity measure of fuzzy sets and their relations. Fuzzy Sets and Systems, 52(3) (1992), 305-318. doi:10.1016/0165-0114(92)90239-Z.

[31] L.A. Zadeh. Fuzzy Sets. Information and Control, 8(3) (1965), 338-353. doi:10.1016/ S0019-9958(65)90241-X.

[32] B.L. Zhang, H. Zhang \& S.S. Ge. Face Recognition by Applying Wavelet Subband Representation and Kernel Associative Memory. IEEE Transactions on Neural Networks, 15(1) (2004), 166-177. 